\title{
Stochastic Energetics of Quantum Transport
}

\author{
Pulak Kumar Ghosh and Deb Shankar Ray* \\ Indian Association for the Cultivation of Science, Jadavpur, Kolkata 700 032, India
}

\begin{abstract}
We examine the stochastic energetics of directed quantum transport due to rectification of nonequilibrium thermal fluctuations. We calculate the quantum efficiency of a ratchet device both in presence and absence of an external load to characterize two quantifiers of efficiency. It has been shown that the quantum current as well as efficiency in absence of load (Stokes efficiency) is higher as compared to classical current and efficiency, respectively, at low temperature. The conventional efficiency of the device in presence of load on the other hand is higher for a classical system in contrast to its classical counterpart. The maximum conventional efficiency being independent of the nature of the bath and the potential remains the same for classical and quantum systems.
\end{abstract}

PACS numbers: 05.40.-a, 05.60.Gg

\section{INTRODUCTION}

Forced thermal ratchet has been the paradigm for rectification of non-equilibrium fluctuations 1, 2, 3, 4] for usable work. In the simplest possible terms it represents a Brownian particle in a periodic potential under overdamped condition which exhibits a net drift provided the system is subjected to an external force with zero mean and with sufficient correlation so that the detailed balance is lost and the symmetry of the device is broken. Over the years considerable attention has been devoted to this area to understand functioning of molecular motors active in muscle contraction [5, 6, 6, , 8], useful separation of particle [9] theoretical issues involving second law 10, 11, 12, 13] and many other aspects 14, 15]. Since a ratchet device is a typical machine which works at a mesoscopic level in converting heat drawn from nonequilibrium fluctuations into work, attempts have been made to quantify the efficiency of such a machine. For example, Sekimoto 13] has proposed a method for studying several variants of thermal ratchet model analyzed also by others [16]. Magnasco [4] has considered a Sziland's heat engine and suggested an expression for net power consumed by such an engine. The work of Julicher et al [3] had provided the estimate of total energy consumption. An interesting generalization of definition of efficiency had been proposed by Derényi et al [24] for motors without load.

We address in this paper the problem of stochastic energetics of a forced thermal ratchet in a quantum mechanical context. A Brownian particle being a microscopic object, the quantum effect is likely to be significant in appropriate situation, e. g, in the transport of quantum particles in quantum wires, superionic 17, 18, 19] conductors and in other nanodevices [20], particularly at low temperature and other important issues 21, 22, 23. . To this end a number of attempts on quantum ratchet device have been made. Reimann et al [26] investigated adia-

*Email Address: pcdsr@mahendra.iacs.res.in batically rocked ratchet system to show that quantum corrections enhance classical transport at low temperature. Two models of quantum ratchet have also been proposed by Yukawa et al [27]. Based on the perturbative approach Scheidl and Vinokur 28] have investigated quantum Brownian motor in ratchet potentials to identify the characteristic scales of response functions of the system. Carlo et al 29] have studied a typical model quantum chaotic dissipative ratchet to analyze the directed transport from a quantum strange attractor. Keeping in view of this development we note that although quantum ratchet device has been the object of interest for some time, its efficiency (i. e., quantum efficiency) has largely remained unexplored. Based on the quantum Langevin equation which implies an interplay between several forces we analyze here the energetics of directed transport by taking into consideration of how the transducer which characterizes the state of system mediates the energy among the basic components of a forced ratchet, i. e., the external system, the load, and the heat bath. It also important that frictional dissipative energy in course of directed motion must also be counted as a part of expenditure of useful energy for rectification of Brownian motion. This implies that one can also envisage a kind of Stokes efficiency in absence of load. We take into account of these considerations in our exploration of quantum energetics in presence and absence of load to characterize two distinct quantifiers of efficiency in a quantum ratchet device. A relevant pertinent point that needs attention in this context is that although quantization, in principle, adds new elements into the theory, it is important that quantization must not break the symmetry of the device, i. e., it should not create a new load or tilt on the potential or break the detailed balance. Secondly, forcing must be unbiased so that after appropriate averaging over time or ensemble, no directional component should appear as a fictitious drift. With these considerations for thermodynamic consistency we analyze the efficiency and current generation in a quantum ratchet in relation to total consumption of energy and dissipation both in presence and absence of external load. 


\section{A QUANTUM DYNAMICS IN A SPATIALLY PERIODIC POTENTIAL AT EQUILIBRIUM}

We consider a particle of mass $m$ moving in a periodic classical potential $V(x)$. The particle is coupled to a set of harmonic oscillators of unit mass acting as a bath. This is represented by the following systemreservoir Hamiltonian 30, 31.

$$
\hat{H}=\frac{\hat{p}^{2}}{2 m}+V(\hat{x})+\sum_{j=1}^{N}\left\{\frac{\hat{p}_{j}^{2}}{2}+\frac{1}{2} \kappa_{j}\left(\hat{q}_{j}-\hat{x}\right)^{2}\right\}
$$

Here $\hat{x}$ and $\hat{p}$ are the coordinate and momentum operators of the particle and $\left\{\hat{q}_{j}, \hat{p}_{j}\right\}$ are the set of coordinate and momentum operators for the reservoir oscillators coupled linearly through the coupling constants $\kappa_{j}(j=1,2, \ldots)$. For the spatially periodic potential, we have $V(x)=V(x+L)$, where $L$ is the length of the period. The coordinate and momentum operators follow the usual commutation rules $\{\hat{x}, \hat{p}\}=i \hbar$ and $\left\{\hat{q}_{i}, \hat{p}_{j}\right\}=i \hbar \delta_{i j}$. Eliminating the bath degrees of freedom in the usual way we obtain the operator Langevin equation for the particle

$$
m \ddot{\hat{x}}+\int_{0}^{t} d t^{\prime} \gamma\left(t-t^{\prime}\right) \dot{\hat{x}}\left(t^{\prime}\right)+V^{\prime}(\hat{x})=\hat{\Gamma}(t)
$$

(Overdots refers to differentiation with respect to time $t$ ) where noise operator $\hat{\Gamma}(t)$ and the memory kernel are given by

$$
\hat{\Gamma}(t)=\sum_{j}\left[\left\{\hat{q}_{j}(0)-\hat{x}(0)\right\} \kappa_{j} \cos \omega_{j} t+\kappa_{j}^{1 / 2} \hat{p}_{j}(0) \sin \omega_{j} t\right]
$$

and

$$
\gamma(t)=\sum_{j} \kappa_{j} \cos \omega_{j} t
$$

respectively, with $\kappa_{i}=\omega_{j}^{2}$

Following Ref. [39] we then carry out a quantum mechanical average $\langle\ldots\rangle$ over the product separable bath modes with coherent states and the system mode with an arbitrary state at $t=0$ in Eq.(2.2) to obtain a generalized quantum Langevin equation [36, 38, 39] as

$$
m \ddot{x}+\int_{0}^{t} d t^{\prime} \gamma\left(t-t^{\prime}\right) \dot{x}\left(t^{\prime}\right)+V^{\prime}(x)=\Gamma(t)+Q\left(x,\left\langle\delta \hat{x}^{n}\right\rangle\right)
$$

where the quantum mechanical mean value of the position operator $\langle\hat{x}\rangle=x$ and

$$
Q\left(x,\left\langle\delta \hat{x}^{n}\right\rangle\right)=V^{\prime}(x)-\left\langle V^{\prime}(\hat{x})\right\rangle
$$

which by expressing $\hat{x}(t)=x(t)+\delta \hat{x}(t)$ in $V(\hat{x})$ and using a Taylor series expansion around $x$ may be rewritten as

$$
Q\left(x,\left\langle\delta \hat{x}^{n}\right\rangle\right)=-\sum_{n \geq 2} \frac{1}{n !} V^{n+1}(x)\left\langle\delta \hat{x}^{n}\right\rangle
$$

The above expansion implies that the nonzero anharmonic terms beyond $n \geq 2$ contain quantum dispersions $\left\langle\delta \hat{x}^{n}\right\rangle$. Although we develop this section in general terms, we are specifically concerned here typically with periodic nonlinear potentials of the type $\sin \frac{2 \pi x}{L}$ or $\cos \frac{2 \pi x}{L}$ or their linear combinations and the like which have been used earlier in several contexts. The nonlinearity of the potential is an important source of quantum correction in addition to the quantum noise of the heat bath. The calculation of $Q$ rests on the quantum correction terms $\left\langle\delta \hat{x}^{n}\right\rangle$ which one determines by solving a set of quantum correction equations as given in the next section. Furthermore the c-number Langevin [36, 38, 39] force is given by

$\Gamma(t)=\sum_{j}\left[\left\langle\hat{q}_{j}(0)\right\rangle-\langle\hat{x}(0)\rangle \kappa_{j} \cos \omega_{j} t+\kappa_{j}^{1 / 2} \hat{p}_{j}(0) \sin \omega_{j} t\right]$

which must satisfy noise characteristics of the bath at equilibrium,

$$
\begin{aligned}
\langle\Gamma(t)\rangle_{S} & =0 \\
\left\langle\Gamma(t) \Gamma\left(t^{\prime}\right)\right\rangle_{S} & =\frac{1}{2} \sum_{j} \kappa_{j} \hbar \omega_{j}\left(\operatorname{coth} \frac{\hbar \omega_{j}}{2 k T}\right) \cos \omega_{j}\left(t-t^{\prime}\right)
\end{aligned}
$$

Eq.(2.10) expresses the quantum fluctuation-dissipation relation. The above conditions $(2.9-2.10)$ can be fulfilled provided the initial shifted co-ordinates $\left\{\left\langle\hat{q}_{j}(0)\right\rangle-\langle\hat{x}(0)\rangle\right\}$ and momenta $\left\langle\hat{p}_{j}(0)\right\rangle$ of the bath oscillators are distributed according to the canonical thermal Wigner distribution [40, 41] of the form

$$
\begin{aligned}
& P_{j}\left(\left[\left\langle\hat{q}_{j}(0)\right\rangle-\langle\hat{x}(0)\rangle\right],\left\langle\hat{p}_{j}(0)\right\rangle\right) \\
= & N \exp \left\{-\frac{\frac{1}{2}\left\langle\hat{p}_{j}(0)\right\rangle^{2}+\frac{1}{2} \kappa_{j}\left[\left\langle\hat{q}_{j}(0)\right\rangle-\langle\hat{x}(0)\rangle\right]^{2}}{\hbar \omega_{j}\left[n\left(\omega_{j}\right)+\frac{1}{2}\right]}\right\}
\end{aligned}
$$

so that the statistical averages $\langle\ldots\rangle_{s}$ over the quantum mechanical mean value $O$ of the bath variables are defined as

$$
\left\langle O_{j}\right\rangle_{s}=\int O_{j} P_{j} d\left\langle\hat{p}_{j}(0)\right\rangle d\left\{\left\langle\hat{q}_{j}(0)\right\rangle-\langle\hat{x}(0)\rangle\right\}
$$

Here $n(\omega)$ is given by Bose-Einstein distributions $\left(e^{\frac{\hbar \omega}{k T}}-\right.$ $1)^{-1} . P_{j}$ is the exact solution of Wigner equation for harmonic oscillator [4, 41] and forms the basis for description of the quantum noise characteristics of the bath kept in thermal equilibrium at temperature $T$. In the continuum limit the fluctuation-dissipation relation (2.10) can be written as

$$
\begin{aligned}
& \left\langle\Gamma(t) \Gamma\left(t^{\prime}\right)\right\rangle \\
= & \frac{1}{2} \int_{0}^{\infty} d \omega \kappa(\omega) \rho(\omega) \hbar \omega \operatorname{coth}\left(\frac{\hbar \omega}{2 k T}\right) \cos \omega\left(t-t^{\prime}\right)
\end{aligned}
$$


where we have introduced the density of the modes $\rho(\omega)$. Since we are interested in the Markovian limit in the present context, we assume $\kappa(\omega) \rho(\omega)=\frac{2}{\pi} \gamma$, Eq. (2.13) then yields

$$
\left\langle\Gamma(t) \Gamma\left(t^{\prime}\right)\right\rangle=2 D_{q} \delta\left(t-t^{\prime}\right)
$$

with

$$
D_{q}=\frac{1}{2} \gamma \hbar \omega_{0} \operatorname{coth} \frac{\hbar \omega_{0}}{2 k T}
$$

(The passage from Eq.(2.13) to Eq.(2.14) is given in the appendix A.)

$\omega_{0}$ refers to static frequency limit. Furthermore from Eq.(2.4) in the continuum limit we have

$$
\gamma\left(t-t^{\prime}\right)=\gamma \delta\left(t-t^{\prime}\right)
$$

$\gamma$ is the dissipation constant in the Markovian limit. In this limit Eq. (2.5) therefore reduces to

$$
m \ddot{x}+\gamma \dot{x}+V^{\prime}(x)=\Gamma(t)+Q\left(x,\left\langle\delta \hat{x}^{n}\right\rangle\right)
$$

It is useful to work with dimensionless variables for the present problem to keep track of the relations between the scales of energy, length and time. The period $L$ of the periodic potential $V(x)$ determines in a natural way the characteristic length scale of the system. Therefore the position of the Brownian particle is scaled as

$$
\bar{x}=x / L
$$

Next we consider the timescales of the system. In absence of the potential and the noise term the velocity of the particle $\dot{x}(t) \sim \exp \left(-t / \tau_{L}\right)$ with $\tau_{L}=m / \gamma$, which represents the correlation time scale of the velocity the Brownian particle. To identify the next characteristic time $\tau_{0}$ we consider the deterministic overdamped motion due to the potential as $\gamma \frac{d x}{d t}=-\frac{d V(x)}{d x}$. Then $\tau_{0}$ is determined from $\gamma \frac{L}{\tau_{0}}=-\frac{\Delta V}{L}$ as $\tau_{0}=\frac{\gamma L^{2}}{\Delta V}$ where $\Delta V$ is the barrier height of the original potential. Hence time is scaled as $\bar{t}=\frac{t}{\tau_{0}}$. Furthermore the potential, the noise and the quantum correction terms are re-scaled as $\bar{V}(\bar{x})=V(x) / \Delta V, \bar{\Gamma}(\bar{t})=\Gamma(t) /(\Delta V / L)$ and $Q /(\Delta V / L)$, respectively.

Hence dimensionless quantum Langevin equation reads as

$$
\mu^{*} \ddot{\bar{x}}+\dot{\bar{x}}=\bar{f}(\bar{x})+\bar{\Gamma}(\bar{t})
$$

Here over-dot(.) refers to differentiation with respect to scaled time $t$. Dimensionless mass $\mu^{*}=\frac{m}{\gamma \tau_{0}}=\frac{\tau_{L}}{\tau_{0}}$ and

$$
\bar{f}(\bar{x})=-\bar{V}^{\prime}(\bar{x})+\bar{Q}\left(\bar{x}, \overline{\left\langle\delta \hat{x}^{n}\right\rangle}\right)
$$

The noise properties of the quantum bath are then rewritten as

$$
\langle\overline{\Gamma(\bar{t})}\rangle_{s}=0
$$

$$
\left\langle\overline{\Gamma(\bar{t})} \overline{\Gamma\left(\bar{t}^{\prime}\right)}\right\rangle_{s}=2 \bar{D}_{q} \delta\left(\bar{t}-\bar{t}^{\prime}\right)
$$

where

$$
\bar{D}_{q}=\frac{\frac{1}{2} \hbar \omega_{0} \operatorname{coth} \frac{\hbar \omega_{0}}{2 k T}}{\Delta V}
$$

From now onwards we drop the over-bars from all parameters and variables for simplicity. It may be shown 36, 37. that quantum stochastic dynamics Eq. (2.18) does not generate drift motion to a preferential direction. Since the quantum correction $Q\left(x,\left\langle\delta \hat{x}^{n}\right\rangle\right)$, as expected, can not break the detailed balance in the quantum system, nor the symmetry of the potential. This conclusion is an important check of the present formalism for a correct description of the equilibrium and thermodynamic consistency.

\section{QUANTUM TRANSPORT INDUCED BY ZERO MEAN EXTERNAL FLUCTUATION}

\section{A. General features}

Since equilibrium thermal fluctuations due to heat bath can not break detailed balance in the quantum stochastic dynamics, we introduce an external derive with zero mean and with sufficient correlation to generate drift motion on average in one direction. To analyze the energetics of directed quantum transport, we now introduce an external load to work against the global motion of the forced thermal ratchet system. From Eq. (2.18) it follows that the dynamics of the particle under overdamped condition is described by the scaled equation (we have dropped the over-bar)

$$
\dot{x}=f(x)+\Gamma(t)+A(t)-\frac{\partial V_{l}}{\partial x}
$$

The quantum mechanical mean of the position operator, $x$ represents the state of the energy transducer, that is the state of the ratchet. $\Gamma(t)$ is the internal quantum noise of the thermal bath with the properties as noted earlier. $A(t)$ is an external field with temporal period $\tau, A(t+\tau)=A(t)$, in the present problem. We consider $A(t)=A_{0} \sin w t$. It is important to note that for a movement of transducer in a preferential direction $A_{0}$ must lie between two threshold values, $\max _{x} f(x)$ and $-\min _{x} f(x)$ [4]. $\frac{\partial V_{l}}{\partial x}=l$, is a load against which transducer performs work. The quantum nature of the problem therefore manifests itself in two ways; first, through quantum 
corrections in $f(x)$ which we consider, in principle, to all orders and secondly in quantum diffusion coefficient $D_{q}$ for the noise of the bath.

The Fokker-Planck equation corresponding to Eq. (3.1) is given by

$$
\frac{\partial P(x, t)}{\partial t}=-\frac{\partial J(x, t)}{\partial x}
$$

where

$$
J(x, t)=-D_{q} \frac{\partial P(x, t)}{\partial x}+[f(x)+A(t)-l] P(x, t)
$$

If forcing frequency is very low, there is enough time for the system to reach the steady state during the period $\tau$ and the above equation can be solved analytically for $J$ as a function of $A$, using period boundary and normalization conditions

$$
P(x+1)=P(x) \quad ; \quad \int_{c}^{c+1} P(x) d x=1 \quad ;
$$

We then obtain

$$
=\frac{J(A)}{N\left[\{\exp [\psi(1)]\}\left[\int_{0}^{1} \exp [\psi(x)] d x-C_{2}\right]+C_{2} \exp [\psi(0)]\right]}
$$

where

$$
\begin{gathered}
N=\frac{1}{D_{q}} \int_{0}^{1} \exp [\psi(x)] d x \\
C_{2}=\frac{\int_{0}^{1} \exp [\psi(x)] d x \int_{0}^{x} d y \exp [\psi(y)]}{\int_{0}^{1} \exp [\psi(x)] d x} \\
\psi(x)=\int_{c}^{x} \frac{f(y)+A-l}{D_{q}} d y ; \quad \psi(L)=\int_{c}^{1} \frac{f(x)+A-l}{D_{q}} d x
\end{gathered}
$$

The average current over a forcing period is given by

$$
J_{a v}=\frac{1}{\tau} \int_{0}^{\tau} J(A(t)) d t
$$

Average square wave current of amplitude $A_{0}$ is given by

$$
J_{s q r}=\frac{1}{2}\left[J\left(A_{0}\right)+J\left(-A_{0}\right)\right]
$$

We now proceed to analyze the current under nonequilibrium condition and the related quantum effects. One of the prime quantities for this analysis is the potential $V(x)$ or the corresponding force term $f(x)$ given by

$$
\begin{aligned}
f(x) & =-\left[V^{\prime}(x)-Q\left(x,\left\langle\delta \hat{x}^{n}\right\rangle\right)\right] \\
& =-\frac{\partial}{\partial x}\left[V(x)+\sum_{n \geq 2} \frac{1}{n !} V^{n}(x)\left\langle\delta \hat{x}^{n}\right\rangle\right]
\end{aligned}
$$

The quantum correction terms can be determined as follows. We return to the operator equation (2.2) and put $\hat{x}(t)=x(t)+\delta \hat{x}(t)$ and $\hat{p}(t)=p(t)+\delta \hat{p}(t)$ where $x(t)=\langle\hat{x}(t)\rangle$ and $p(t)=\langle\hat{p}(t)\rangle$ are the quantum mechanical mean values of the operators $\hat{x}$ and $\hat{p}$ respectively. By construction $[\delta \hat{x}, \delta \hat{p}]=i \hbar$ and $\langle\delta \hat{x}\rangle=\langle\delta \hat{p}\rangle=0$. We then obtain the quantum correction equation

$$
\begin{gathered}
m \delta \ddot{\hat{x}}+\int_{0}^{t} d t^{\prime} \gamma\left(t-t^{\prime}\right) \delta \dot{\hat{x}}\left(t^{\prime}\right)+V^{\prime \prime}(x) \delta \hat{x} \\
+\sum_{n \geq 2} \frac{1}{n !} V^{n+1}(x)\left(\delta \hat{x}^{n}-\left\langle\delta \hat{x}^{n}\right\rangle\right)=\hat{\Gamma}(t)-\Gamma(t)
\end{gathered}
$$

Again in the overdamped limit we discard the inertial term $m \delta \ddot{\hat{x}}$. We then perform a quantum mechanical average with initial product separable coherent states of the oscillators of the bath only to get rid of the internal noise term and to obtain the reduced operator equation for the system as

$$
\gamma \delta \dot{\hat{x}}+V^{\prime \prime}(x) \delta \hat{x}+\sum_{n \geq 2} \frac{1}{n !} V^{n+1}(x)\left(\delta \hat{x}^{n}-\left\langle\delta \hat{x}^{n}\right\rangle\right)=0
$$

With the help of (3.13) we then obtain the equations for $\left\langle\delta \hat{x}^{n}(t)\right\rangle$

$$
\begin{aligned}
\frac{d}{d t}\left\langle\delta \hat{x}^{2}\right\rangle= & \frac{1}{\gamma}\left[-2 V^{\prime \prime}(x)\left\langle\delta \hat{x}^{2}\right\rangle-V^{\prime \prime \prime}(x)\left\langle\delta \hat{x}^{3}\right\rangle\right] \\
\frac{d}{d t}\left\langle\delta \hat{x}^{3}\right\rangle & =\frac{1}{\gamma}\left[-3 V^{\prime \prime}(x)\left\langle\delta \hat{x}^{3}\right\rangle-\frac{3}{2} V^{\prime \prime \prime}(x)\left\langle\delta \hat{x}^{4}\right\rangle\right] \\
& +\frac{3}{2 \gamma} V^{\prime \prime \prime}(x)\left\langle\delta \hat{x}^{2}\right\rangle^{2}
\end{aligned}
$$

and so on. Taking into account of the leading order contribution $\left\langle\delta \hat{x}^{2}\right\rangle$ explicitly we may write (it is easy to observe that each successive order of quantum correction decreases by a factor of $O(1 / \gamma)$ which implies that a leading order contribution is sufficient in the overdamped limit)

$$
d\left\langle\delta \hat{x}^{2}\right\rangle=-\frac{2}{\gamma} V^{\prime \prime}(x)\left\langle\delta \hat{x}^{2}\right\rangle d t
$$

The overdamped deterministic motion gives $\gamma d x=$ $-V^{\prime}(x) d t$ which when used in (3.16) yields after integration

$$
\left\langle\delta \hat{x}^{2}\right\rangle=\Delta_{q}\left[V^{\prime}(x)\right]^{2}
$$

where $\Delta_{q}=\frac{\left\langle\delta \hat{x}^{2}\right\rangle_{x_{c}}}{\left[V\left(x_{c}\right)\right]^{2}}$ and $x_{c}$ is a quantum mechanical mean position at which $\left\langle\delta \hat{x}^{2}\right\rangle$ become minimum, i. e., $\left\langle\delta \hat{x}^{2}\right\rangle_{x_{c}}=\frac{1}{2} \hbar / \omega_{0}, \omega_{0}$ being defined in Eq. (2.15) 
In present problem we consider an asymmetric potential of period $2 \pi$,

$$
V(x)=-\sin x-0.25 \sin 2 x
$$

The reference point $x_{c}$ can be determined by setting $\frac{d\left\langle\delta \hat{x}^{2}\right\rangle}{d x}=0$ and quantum correction up to the leading order and the potential force are given by

$$
Q\left(x,\left\langle\delta \hat{x}^{n}\right\rangle\right)=-\Delta_{q}\left[V^{\prime}(x)\right]^{2}\left[V^{\prime \prime \prime}(x)\right]
$$

and

$$
f(x)=-\left[V^{\prime}(x)+\Delta_{q} V^{\prime \prime \prime}(x)\left[V^{\prime}(x)\right]^{2}\right]
$$

respectively, where $\Delta_{q}=\frac{2 \hbar}{\omega_{0}}$. We now emphasize an important point. If the potential is symmetric, then the quantum correction in Eq. (3.20) is an odd function just as $V^{\prime}(x)$. This implies that quantum correction to classical potential has not destroyed the inversion symmetry of $V(x)$. Thus the approximation in deriving the leading order quantum effect is consistent with symmetry requirement of the problem. It also clear that if potential is periodic then the contribution due to quantum correction to the classical potential i., e., $\int_{0}^{x} Q\left(x,\left\langle\delta \hat{x}^{n}\right\rangle\right) d x$ is a periodic function of $x$. Assuming the form of potential of Eq.(3.18), the expression for quantum correction after properly scaling (as described in the Sec.II) is given by

$$
\begin{aligned}
Q\left(x,\left\langle\delta \hat{x}^{n}\right\rangle\right) & =-\Delta_{q}\left[\cos ^{3} 2 \pi x+0.5 \cos ^{3} 4 \pi x\right. \\
& \left.+3 \cos ^{2} 2 \pi x \cos 4 \pi x+2.25 \cos 2 \pi x \cos ^{2} 4 \pi x\right]
\end{aligned}
$$

Quantum correction of the potential is entirely due to nonlinearity. Physically the correction terms account for the quantum fluctuation or dispersion around the classical path of a dynamical system. In presence of strong dissipation these fluctuations are small since it is wellknown (also follows from analysis of Eqs (3.13 3.17)) that dissipation enhances classicality 32. The role of effective potential of the similar nature which gives rise to leading order quantum correction to classical Langevin force had also been noted earlier, e. g., in the analysis of strong friction limit of quantum stochastic processes etc. [33, 34, 35]. Since the corrections are perturbative in nature they may differ in form but because of nonlinearity of the potential they bear close kinship to each other. We emphasize that the approximate forms of quantum correction must satisfy the basic symmetry requirement, appropriate equilibrium distribution and other thermodynamic consistency condition as pointed out earlier 36, 37]. In Fig.1 we illustrate the variation of current as a function of temperature( $\mathrm{T}$ ) for different values of the amplitude of external derive $\left(A_{0}\right)$. One observes that with increase of $D_{q}$ (proportional to temperature) the magnitude of current increases to a maximum followed by decrease and a current reversal at high temperature. At higher temperature the system is thermalized as a result of which organized motion in a preferential direction decreases and the motion towards the load dominates. For fixed $D_{q}$ with increase of the value of $A_{0}$ the magnitude of the current increases. The effect of quantization of a classical ratchet is shown in Fig.2, where we present a comparison of the current vs temperature profile for the classical and the quantum cases. One observes that at the low temperature region the classical current is significantly lower in magnitude than quantum current and at the higher temperature the effect of quantization becomes insignificant. This may be interpreted in terms of an interplay between quantum diffusion coefficient $D_{q}$ and the potential force term $f(x) . f(x)$ contains quantum correction arising due to nonlinearity of the potential. As temperature $T \rightarrow 0, D_{q}$ approaches to the value $\frac{1}{2} \hbar \omega_{0}$, the vacuum limit in deep tunneling region. The anharmonic terms in $f(x)$ do not contribute significantly. So the integrand in effective potential $\psi(x)$ increases sharply. On the other hand, as temperature increases, $D_{q}$ increases and also $D_{q}$ and $f(x)$ compete with each other to merge quantum current to its classical counterpart.

\section{B. Energetics of non-equilibrium fluctuation induced quantum transport}

Efficiency of the ratchet device is an important physical quantity that quantifies the energetics of non-equilibrium fluctuations in the transport processes. Depending on the degree and presence of an external load two distinct approaches have been advocated. It has been shown that although in many widely accepted cases efficiency is measured by applying the constant external force, there are situations, where molecular motors are designed not to pull loads (e. g., protein transport within a cell). In such cases a minimum energy input is required to move a particle in a viscous medium. We therefore discuss the two different situations separately.

\section{Conventional efficiency in presence of an external load}

To discuss the energetics of quantum transport induced by zero mean external derive we consider the energy transducer which interacts with the external derive and the load so that the potential takes the following form

$$
U(x, t)=V(x)-\int d x Q\left(x,\left\langle\delta \hat{x}^{n}\right\rangle\right)+A(t) x+l x
$$

where $V(x)$ is the classical potential, second term represents the quantum corrections due to nonlinearity of the classical potential and last two terms are due to external system and the load, respectively. The interaction of transducer with heat bath is assumed to be stochastic, as usual. Thus for the movement of transducer from $x_{i}\left(t_{i}\right) \rightarrow x_{f}\left(t_{f}\right)$ the total potential energy change $(\Delta U)$ and dissipation energy $\left(E_{d}\right)$, during the period $t_{i}<t<t_{f}$ are formally given by 13 ] 


$$
\Delta U=U\left(x_{f}\left(t_{f}\right), t_{f}\right)-U\left(x_{i}\left(t_{i}\right), t_{i}\right)
$$

and

$$
E_{d}=\int_{t_{i}}^{t_{f}}[-\dot{x}+\Gamma(t)] d x(t)=\int_{t_{i}}^{t_{f}}-\left[\frac{\partial U(x, t)}{\partial x}\right] d x(t)
$$

respectively.

Because of the conservation law, the sum of the potential energy change and dissipation energy must be equal to the total consumption of energy $E_{c}\left(\triangle U+E_{d} \equiv E_{c}\right)$, due to the external system $A(t)$.

$$
E_{c}=\int_{t_{i}}^{t_{f}} \frac{\partial U(x, t)}{\partial t} d t
$$

In the present case the external system is a periodic function of time, so that the ensemble average of total consumption energy $\left(E_{c}\right)$ and dissipation energy $\left(E_{d}\right)$ is given by

$$
\begin{aligned}
\left\langle E_{c}\right\rangle & =\int_{t_{i}}^{t_{f}} d t \int_{\text {space }} \frac{\partial U(x, t)}{\partial t} P(x, t) d x(t) \\
& =\int_{t_{i}}^{t_{f}} d t \int_{\text {space }} A(t) J(A(t)) d x(t)
\end{aligned}
$$

and

$$
\left\langle E_{d}\right\rangle=\int_{t_{i}}^{t_{f}} d t \int_{\text {space }} d x\left[-\frac{\partial U(x, t)}{\partial x}\right] J(A(t))
$$

respectively. For the square wave with the amplitude $A_{0}$,

$$
\begin{aligned}
\left\langle\left(E_{c}\right)_{s q r}\right\rangle & =\frac{1}{2} A_{0}\left[J\left(A_{0}\right)-J\left(-A_{0}\right)\right] \\
\left\langle\left(E_{d}\right)_{s q r}\right\rangle & =\frac{1}{2} A_{0}\left\{J\left(A_{0}\right)-J\left(-A_{0}\right)\right\} \\
& -\frac{1}{2} l\left\{J\left(A_{0}\right)+J\left(-A_{0}\right)\right\}
\end{aligned}
$$

Hence the work, that the ratchet system extracts from the external system $A(t)$ is given by

$$
\begin{aligned}
\left\langle W_{s q r}\right\rangle & =\left\langle\left(E_{c}\right)_{s q r}\right\rangle-\left\langle\left(E_{d}\right)_{s q r}\right\rangle=\frac{1}{2} l\left[J\left(A_{0}\right)+J\left(-A_{0}\right)\right] \\
& =l \times J_{s q r}
\end{aligned}
$$

So the work extracted from external system is directly proportional to square wave current. The conventional efficiency of the ratchet system is thus calculated on the basis of external load can be written as

$$
\begin{aligned}
\eta & =\frac{l J_{s q r}}{E_{c}} \\
& =\frac{l\left[J\left(A_{0}\right)+J\left(-A_{0}\right)\right]}{A_{0}\left[J\left(A_{0}\right)-J\left(-A_{0}\right)\right]}
\end{aligned}
$$

We now numerically illustrate the behavior of efficiency of the quantum ratchet system as given above. The effect of quantization of the reservoir is apparent in Fig.3 in the variation of efficiency $(\eta)$ as a function of temperature for different values of amplitude $\left(A_{0}\right)$ of the external periodic system. The efficiency is a decreasing function of temperature for any value of $A_{0}$ and it decreases with increase of $A_{0}$ for a fixed value of temperature. The distinctive behavior of efficiency of a quantum system is evident from the nature of quantum current that can attain a maximum value for a finite temperature. On the other hand the maximum efficiency is realized at the zero strength of thermal fluctuation. It is thus apparent that the equilibrium fluctuation due to thermal heat bath is an hindrance for efficient extraction of useful work from non-equilibrium fluctuations. To have a closer look at the behavior of efficiency we present in Fig.4 the dissipation energy and total consumption energy as a function of temperature. At very low temperature the energy loss due to dissipation $\left(E_{d}\right)$ during the movement of energy transducer is small compared to the total energy consumed from the external system, because for a finite net displacement $x_{i} \rightarrow x_{f}$ it covers minimum path at low thermal fluctuation. On the other hand at high temperature the path of energy transducer is more chaotic. So for a finite net displacement it covers maximum path and loses a greater amount energy due to dissipation. With increase of the temperature dissipation energy and total energy consumption from external system both are increased and the difference between two energies $\left(E_{d}\right.$ and $E_{c}$ ) become insignificant at higher temperature.

The condition for maximum conventional efficiency can be realized by rearranging Eq. (3.31) as a function of $\frac{J\left(-A_{0}\right)}{J\left(A_{0}\right)}$

$$
\eta=\frac{l}{A_{0}}\left(1-\frac{2\left|\frac{J\left(-A_{0}\right)}{J\left(A_{0}\right)}\right|}{1+\left|\frac{J\left(-A_{0}\right)}{J\left(A_{0}\right)}\right|}\right)
$$

In the limit $\left|\frac{J\left(-A_{0}\right)}{J\left(A_{0}\right)}\right| \rightarrow 0$, the maximum efficiency of the energy transform for a given load and force amplitude is given by (the limit can be achieved by suitable adjustment of parameters)

$$
\eta_{\max }=\frac{l}{A_{0}}
$$

Now we have two important conclusions regarding the maximum efficiency of a ratchet system, (i) it is a simple ratio of load to a parameter of external system (strength 
of external system) and it is independent of the characteristics of the bath. (ii) $\eta_{\max }$ being independent of the nature of the bath and the system potential, is same both in quantum and classical systems.

In Fig. 5 we compare the conventional efficiency vs temperature profile for the classical and the quantum cases for different values of $A_{0}$. We observe that the efficiency of quantum ratchet is significantly lower than classical one and the difference becomes insignificant at higher temperature. Since the vacuum fluctuations tend to be effective in the quantum system as one approaches the zero temperature limit, the transducer loses a higher amount of dissipation energy than the classical one.

\section{Efficiency in absence of an external load and generalized efficiency}

We now consider the situation where the motor works without any external load. The task is not only to translocate the motor over a distance $L$ but also to do this with a given average velocity it must work against the viscous force $\gamma\langle v\rangle$. Now replacing the load by $\gamma\langle v\rangle$ we can define an efficiency (Stokes efficiency)

$$
\eta_{S}=\frac{\gamma\langle v\rangle^{2}}{E_{c}}
$$

By combining the contribution due to (3.31) and (3.34), it is possible to define further a generalized efficiency for the quantum system

$$
\eta_{G}=\frac{l J_{s q r}+\gamma\langle v\rangle^{2}}{E_{c}}
$$

The above expression is the quantum generalization of the classical generalized efficiency as given earlier by Suzuki and Munakata [25] and Derényi et al 24]. This account for both the work that the motor performs against the external load $l$ as well as the work that is necessary to move the particle over a given distance in a viscous environment at the average velocity $\langle v\rangle$.

In Fig.6 we the present variation of Stokes efficiency $\eta_{S}$ as a function of temperature. It is important to observe that efficiency reaches a maximum at a particular temperature both for classical as well as for the quantum case. However, again at low temperature the efficiency of the classical system drops to zero in sharp contrast to quantum case. At high temperature the system however, tends to classical regime as expected.

\section{CONCLUSION}

In this paper we have calculated the efficiency of a forced thermal ratchet in a quantum mechanical context. We have shown that the quantum current is markedly higher as compared to classical current at low temperature while the difference becomes insignificant at higher temperature. In contrast to the behavior of quantum current at low temperature, the conventional efficiency of a classical ratchet in presence of a load is higher at low temperature as compared to its quantum counterpart and again the efficiency in the two cases tends to merge at higher temperature. Furthermore the maximum efficiency is independent of the nature of the system potential and the bath and is thus independent of quantization. We have also examined a quantum version of Stokes efficiency in absence of load where energy due to frictional resistance is considered as a part of expenditure of useful energy. A significant quantum enhancement of Stokes efficiency at low temperature has been observed. The careful consideration of the total energy consumption and dissipation reveals that the generation of higher current and Stokes efficiency may not always imply the higher efficiency of thermal ratchet in a conventional sense although the generic features of the device in its classical and quantum versions remain the same.

\section{Acknowledgments}

Thanks are due to the Council of Scientific and Industrial Research, Govt. of India, for a fellowship (PKG).

\section{APPENDIX A: THE PASSAGE FROM EQ.(2.13)} TO EQ. (2.14)

We start from basic definition [42]

$$
2 D_{q}=\frac{1}{2 \Delta t} \int_{t}^{t+\Delta t} d t_{1} \int_{t}^{t+\Delta t} d t_{2}\left\langle\Gamma\left(t_{1}\right) \Gamma\left(t_{2}\right)\right\rangle_{s}
$$

Using Eq.(2.13) in Eq.(1) yields

$$
\begin{aligned}
2 D_{q} & =\frac{1}{2 \Delta t} \int_{0}^{\infty} d \omega \kappa(\omega) \rho(\omega) \hbar \omega \operatorname{coth}\left(\frac{\hbar \omega}{2 k T}\right) \\
& \times \int_{t}^{t+\Delta t} d t_{1} \int_{t}^{t+\Delta t} d t_{2} \cos \omega\left(t_{1}-t_{2}\right)
\end{aligned}
$$

Explicit integration over time gives

$$
2 D_{q}=\frac{1}{2 \Delta t} \int_{0}^{\infty} d \omega \kappa(\omega) \rho(\omega) \hbar \omega \operatorname{coth}\left(\frac{\hbar \omega}{2 k T}\right) \quad I(\omega, \Delta t)
$$

where

$$
I(\omega, \Delta t)=\frac{4}{w^{2}} \sin ^{2} \frac{\omega \Delta t}{2}
$$

Putting $\kappa(\omega) \rho(\omega)=\frac{2}{\pi} \gamma$, we obtain

$$
2 D_{q}=\frac{\gamma}{\Delta t \pi} \int_{0}^{\infty} d \omega \hbar \omega \operatorname{coth}\left(\frac{\hbar \omega}{2 k T}\right) \frac{\sin ^{2} \frac{\omega \Delta t}{2}}{\left(\frac{\omega}{2}\right)^{2}}
$$

Following Louisell[42] we have under Markovian condition, the correlation time $\tau_{c} \ll \Delta t$, the coarsegrain time(over which the probability distribution function evolves). Thus as $\Delta t \rightarrow \infty$ (in scale of $\tau_{c}$ which goes 
to zero) the function $\frac{\sin ^{2} \frac{\omega \Delta t}{2}}{\left(\frac{\omega}{2}\right)^{2}}$ oscillates violently so that one takes the slowly varying quantity $\left[\hbar \omega \operatorname{coth} \frac{\hbar \omega}{2 k T}\right]$ out of the integration over frequency with an average value $\hbar \omega_{0} \operatorname{coth} \frac{\hbar \omega_{0}}{2 k T}, \omega_{0}$ be an average static frequency. Since the integral $\int_{\infty}^{\infty} \frac{\sin ^{2} x \Delta t}{x^{2}} d x=\pi \Delta t$ it follows immediately from Eq. (5)

$$
2 D_{q}=\gamma \hbar \omega_{0} \operatorname{coth}\left(\frac{\hbar \omega_{0}}{2 k T}\right)
$$

as given in Eq.[2.15]. Again starting from Eq.(2.13), we use the same argument as before to have

$$
\begin{aligned}
\left\langle\Gamma\left(t_{1}\right) \Gamma\left(t_{2}\right)\right\rangle_{s} & =\frac{1}{2} \int_{0}^{\infty} d \omega \kappa(\omega) \rho(\omega) \hbar \omega \\
& \times \operatorname{coth}\left(\frac{\hbar \omega}{2 k T}\right) \cos \omega\left(t_{1}-t_{2}\right)
\end{aligned}
$$

and we use

$$
\int_{0}^{\infty} d \omega \cos \omega \tau=\pi \delta(\tau)
$$

to obtain

$$
\begin{aligned}
\left\langle\Gamma\left(t_{1}\right) \Gamma\left(t_{2}\right)\right\rangle_{s} & =\frac{1}{2} \int_{0}^{\infty} d \omega\left[\frac{2}{\pi} \gamma\right] \hbar \omega \\
& \times \operatorname{coth}\left(\frac{\hbar \omega}{2 k T}\right) \cos \omega\left(t_{1}-t_{2}\right) \\
& =\frac{\gamma \hbar \omega_{0}}{\pi} \operatorname{coth}\left(\frac{\hbar \omega_{0}}{2 k T}\right) \pi \delta\left(t_{1}-t_{2}\right) \\
& =\gamma \hbar \omega_{0} \operatorname{coth}\left(\frac{\hbar \omega_{0}}{2 k T}\right) \delta\left(t_{1}-t_{2}\right)
\end{aligned}
$$

Therefore from Eq.(6) and Eq.(7) we have

$$
\left\langle\Gamma\left(t_{1}\right) \Gamma\left(t_{2}\right)\right\rangle_{s}=2 D_{q} \delta\left(t_{1}-t_{2}\right)
$$

Thus the derivation within Markovian approximation clearly depends on the time scale separation. The results are valid even at absolute zero as emphasized by Louisell.
[1] A. Ajdari and J. Prost, Comptes Rendus Acad. Sci. Paris 315, 1635 (1992); M. Büttiker, Z. Phys B 68, 161 (1987).

[2] M. O. Magnasco, Phys. Rev. Lett. 71, 1477 (1993); P. Reiman, Phys. Reps. 361, 57 (2002);

[3] F. Jülicher and J. Prost, Phys. Rev. Lett. 75, 2618 (1995)

[4] M. O. Magnasco, Euro Phys. Lett. 72, 2656 (1995);

[5] E. H. Sepersu and T. Y. Tsons, J. Membr. Biol. 74, 191 (1983); J. Bio. Chem. 2591477 (1984)

[6] R. D. Vale and F. Oosawa, Adv. Biophys. 26, 97 (1990)

[7] J. Howard, Mechanics of Motor Proteins and the Cytoskeletons (Sinauer Associates, Sunderland 2001).

[8] T. Y. Tsong and R. D. Astumian, Bioelectrochems Bioenergy 211457 (1986); H.V. Westerhoff, T.Y. Tsong, P.B. Chock, Y.D. Chen, and R.D. Astumian, Proc. Natl. Acad. Sci. U.S.A. 83, 4734 (1986).

[9] J. Rousselet, L. Salome, A. Adjari and J. Prost, Nature 370, 446 (1994).

[10] C. Van den Broeck, R. Kawai, and P. Meurs, Phys. Rev. Lett. 93, 090601 (2004).

[11] H. S. Leff and A. F. Rex, Maxwell's Demon, Entropy, Information, Computing (Adam Hilger, Bristol 1990).

[12] N. G. van Kampen, IBM J. Res. Develop. 32, 107 (1988).

[13] K. Sekimoto, J. Phys. Soc. Jap 661234 (1997).

[14] A. M. Glass, D. von der Linde, T. J. Negran, Appl. phys. Lett. 25, 233 (1974).

[15] P. J. Sturman, Photovoltaic and Photo-refractive Effects in Noncentrosymmetric Materials (Gordon and Breach, Philadelphia 1992).

[16] H. Kamegawa, T. Hondou and F. Takagi, Phys. Rev. Lett. 80, 5251 (1998).

[17] P. Brüesch, S. Strässler and H. R. Zeller, Phys. Status. Solidi. (a) 31, 217 (1975); P. Fulde, L. Pietronero, W. R. Schneider and S. Strässler, Phys. Rev. Lett. 35, 1776 (1975).
[18] T. Geisel, in Physics of Superionic Conductor, ed. by M. B. Soloman, Topic Current Phys., Vol. 15 (Springer, Berlin, Heidelberg, N. Y., 1997) p 201

[19] W. Dicterich, P. Fulde and I. Peschel, Adv. Phys. 29527 (1980)

[20] H. Linke, T. E. Humphrey, A. Lfgren, A. O. Sushkov, R. Newbury, R. P. Taylor, and P. Omling, Science 286, 2314 (1999).

[21] J. Łuczka, M. Niemiec and E. Piotrowski, J. Phys. A 26, 4849 (1993).

[22] C. R. Doering, W. Horsthemke and J. Riordan, Phys. Rev. Lett. 72, 2984 (1994).

[23] A. Mielke, Ann. Phys. 4, 476 (1995).

[24] I. Derényi, M. Bier and R. D. Astumian, Phys. Rev. Lett. 83, 903 (1999).

[25] D. Suzuki and T. Munakata Phys. Rev. E 68 , 021906 (2003).

[26] P. Reimann, M. Grifoni, and P. Hänggi, Phys. Rev. Lett. 79, 10 (1997); See also, P. Hänggi, F. Marchesoni and F. Nori, Ann. Phys. (Leipzig) 14, 51 (2005).

[27] S. Yukawa, G. Tatara, M. Kikuchi and H. Matsukawa, Physica B 284, 1896 (2000).

[28] S. Scheidl and V. M. Vinokur, Phys. Rev. B 65, 195305 (2002).

[29] G. G. Carlo, G. Benenti, G. Casati and D. L. Shepelyansky, Phys. Rev. Lett. 94, 164101 (2005).

[30] R. Zwanzig, J. Stat. Phys.9, 215 (1973).

[31] H. Mori, Progr. Theor. Phys. 33, 423 (1965); H. Mori, H. Fujisaka, ibid. 49, 764 (1973), M. Tokuyama, H. Mori, ibid. 55, 411 (1976), 56, 1073 (1976); L. S. Garcia-Colin, J. L. Del Rio, J. Stat. Phys. 16, 235 (1977).

[32] T. Dittrich and R. Graham, Ann. Phys. 200, 363 (1990); G. G. Carlo, G. Benenti, and D. L. Shepelyansky, Phys. Rev. Lett. 95, 164101 (2005).

[33] J. Luczka, R. Rudnicki and P. Hänggi, Physica A 351, 
$60(2005)$

[34] J. Ankerhold, P. Pechukas and H. Grabert Phys. Rev. Lett. 87, 086802 (2001).

[35] J. D. Bao, Phys. Rev. A 65, 052120 (2002).

[36] P. K. Ghosh, D. Barik and D. S. Ray, Phys. Rev. E 71, 041107 (2005).

[37] D. Barik and D. S. Ray, J. Stat. Phys. 120 339(2005).

[38] P. K. Ghosh, D. Barik and D. S. Ray, Phys. Lett. A 342 12 (2005).

[39] D. Banerjee, B. C. Bag, S. K. Banik and D. S. Ray, Phys. Rev. E 65, 021109 (2002).

[40] E. P. Wigner, Phys. Rev. 40, 749 (1932).

[41] M. Hillery, R. F. O'Connell, M. O. Scully and E. P. Wigner, Phys. Reps. 106, 121 (1984).

[42] W. H. Louisell, Quantum Statistical Properties of Radiation, (John Wiley and Sons, New York, 1990);

\section{Figure Captions}

Fig.1. A plot of quantum current vs $T$ for different strength of external periodic force (i) $A_{0}=1.0$ (dotted line), (ii) $A_{0}=1.2$ (solid line), (iii) $A_{0}=1.5$ (dash-dot line) and $l=0.01$.(All the quantities are dimensionless)

Fig.2. A current $J_{\text {sqr }}$ vs temperature $(T)$ plot comparing classical(dashed line and solid line) and quantum (dotted line and dashed dot line) limit for different strength of external periodic force (i) $A_{0}=1.3$ (dotted line and dashed line), (ii) $A_{0}=1.0$ (dashed dot line and solid line) and $l=0.0$.(All the quantities are dimensionless)

Fig.3. A plot of quantum efficiency vs $T$ for different strength of external periodic force (i) $A_{0}=1.0$ (dotted line), (ii) $A_{0}=1.5$ (solid line), (iii) $A_{0}=2.0$ (dashed line) and $l=0.01$.(All the quantities are dimensionless)

Fig.4. A comparison between dissipation energy $E_{d}\left(\right.$ dotted line) and total energy consumption $E_{c}$ (solid line) as a function of temperature for the parameter set $l=0.05$ and $A_{0}=1.0$.(All the quantities are dimensionless)

Fig.5. Conventional efficiency $(\eta)$ vs temperature $(T)$ plot comparing classical(dotted line and solid line) and quantum (dashed line and dashed dot line) limit for different strength of external periodic force (i) $A_{0}=1.3$ ( dotted line and dashed line ), (ii) $A_{0}=1.0$ (solid line and dashed dot line) and $l=0.0$.(All the quantities are dimensionless).

Fig.6. A comparison between classical(dashed dot line and dashed line) and quantum (solid and dotted line) Stokes efficiency for different strength of external periodic force (i) $A_{0}=1.3$ (solid line and dashed dot line), (ii) $A_{0}=1.0$ (dotted line and dashed line) and $l=0.0$.(All the quantities are dimensionless) 


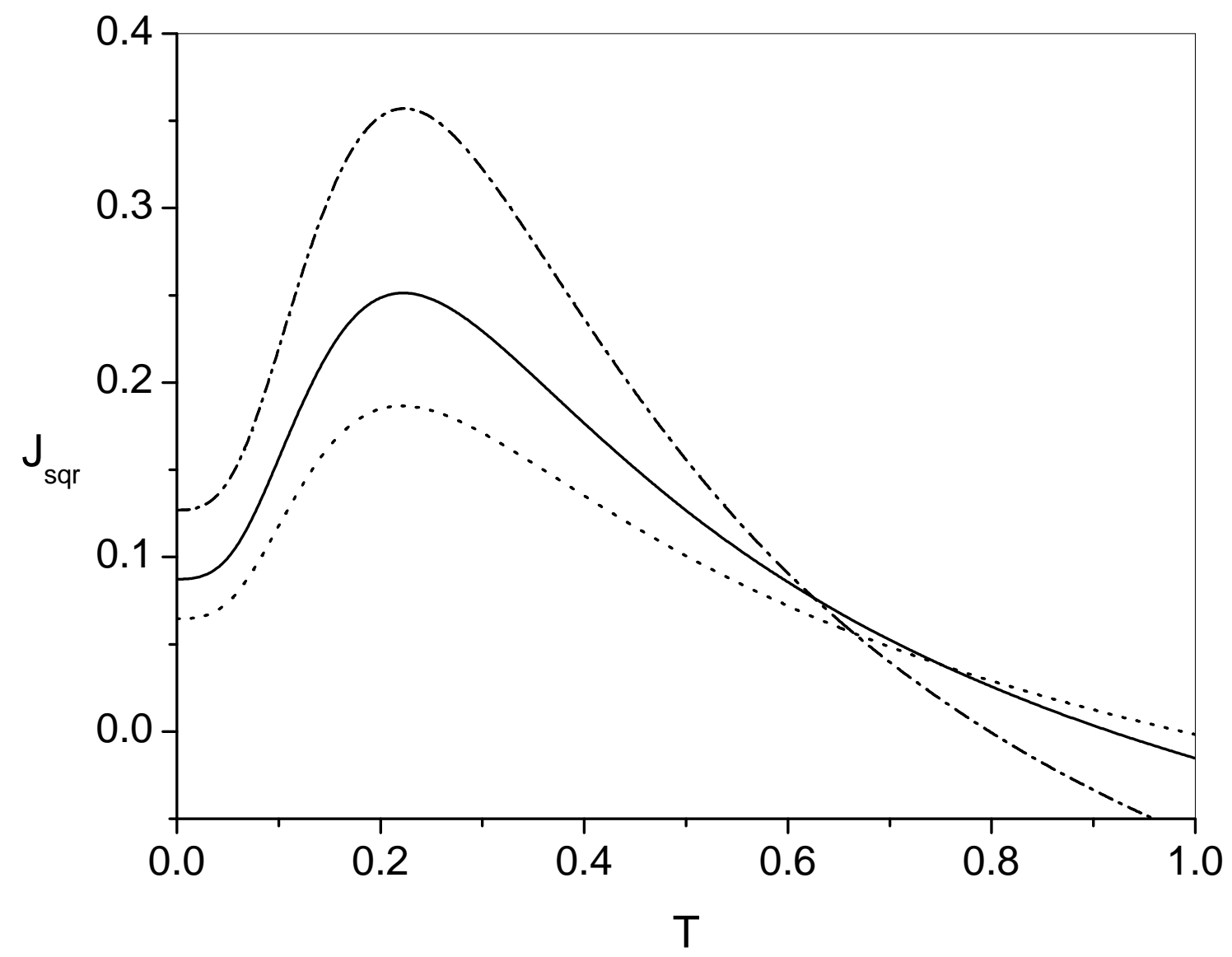

Fig.1

Title: Stochastic Energetics of Quantum Transport Aauthor: Pulak Kumar Ghosh and Deb Shankar Ray 


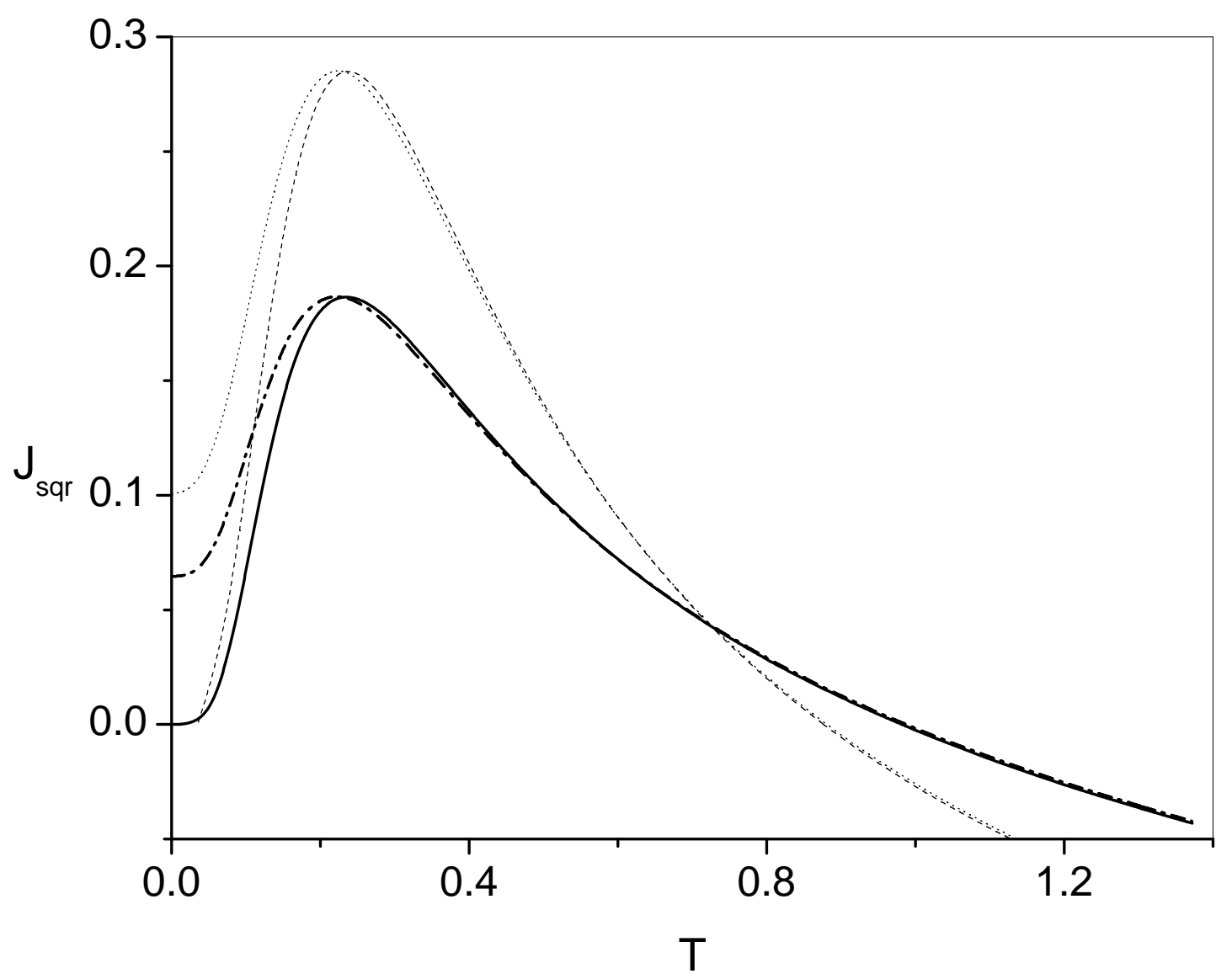

Fig.2

Title: Stochastic Energetics of Quantum Transport Aauthor: Pulak Kumar Ghosh and Deb Shankar Ray 


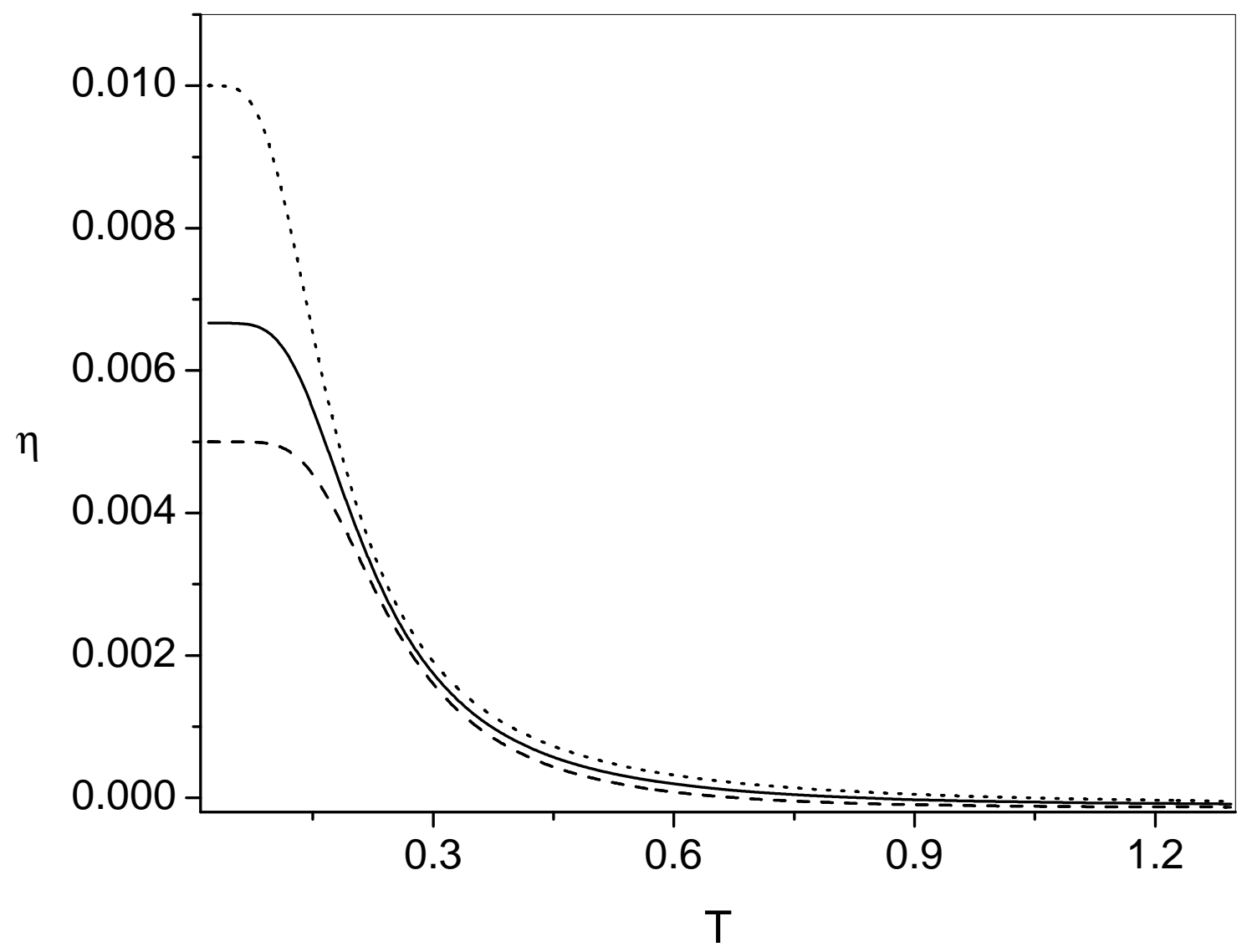

Fig.3 


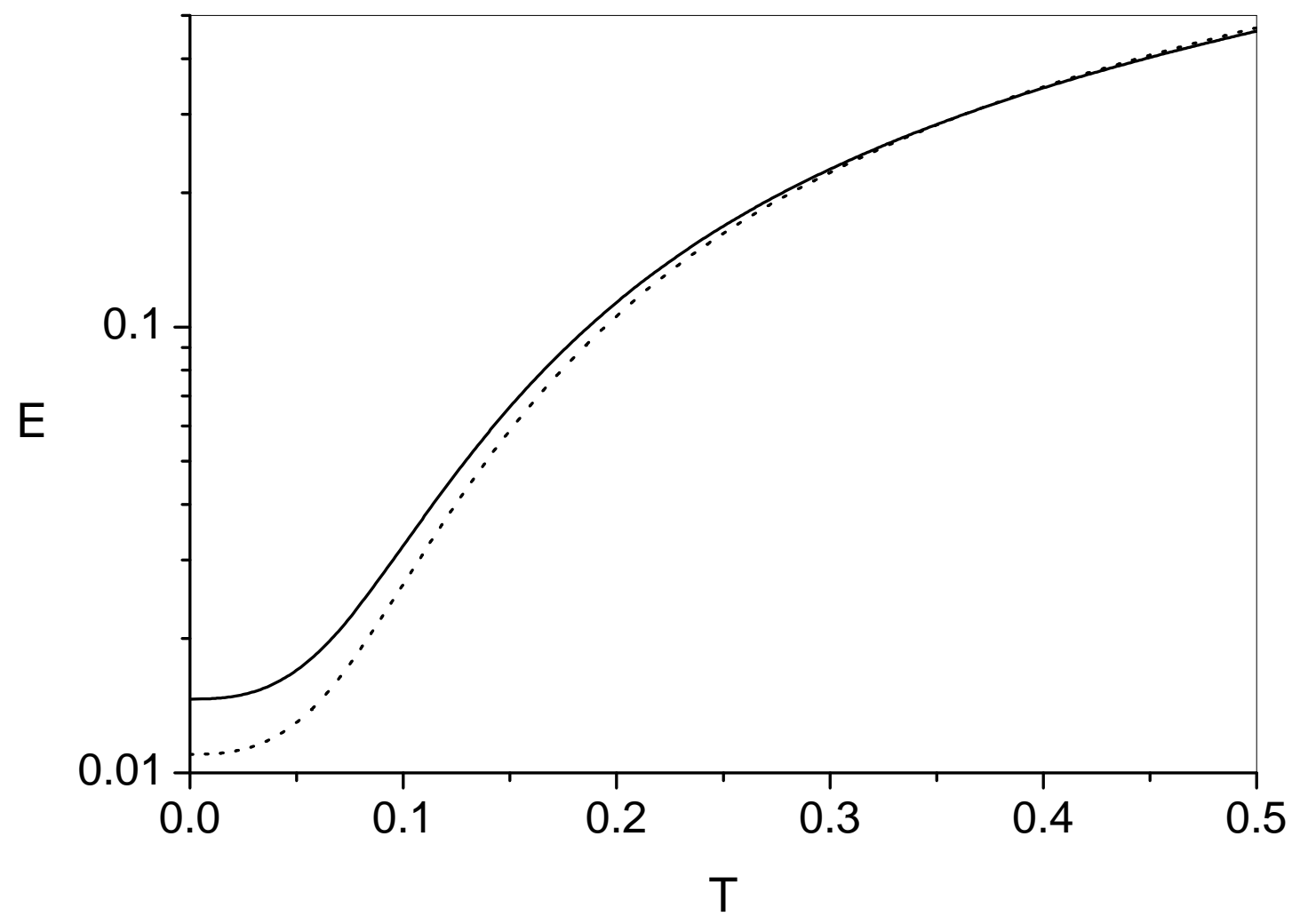

Fig.4 


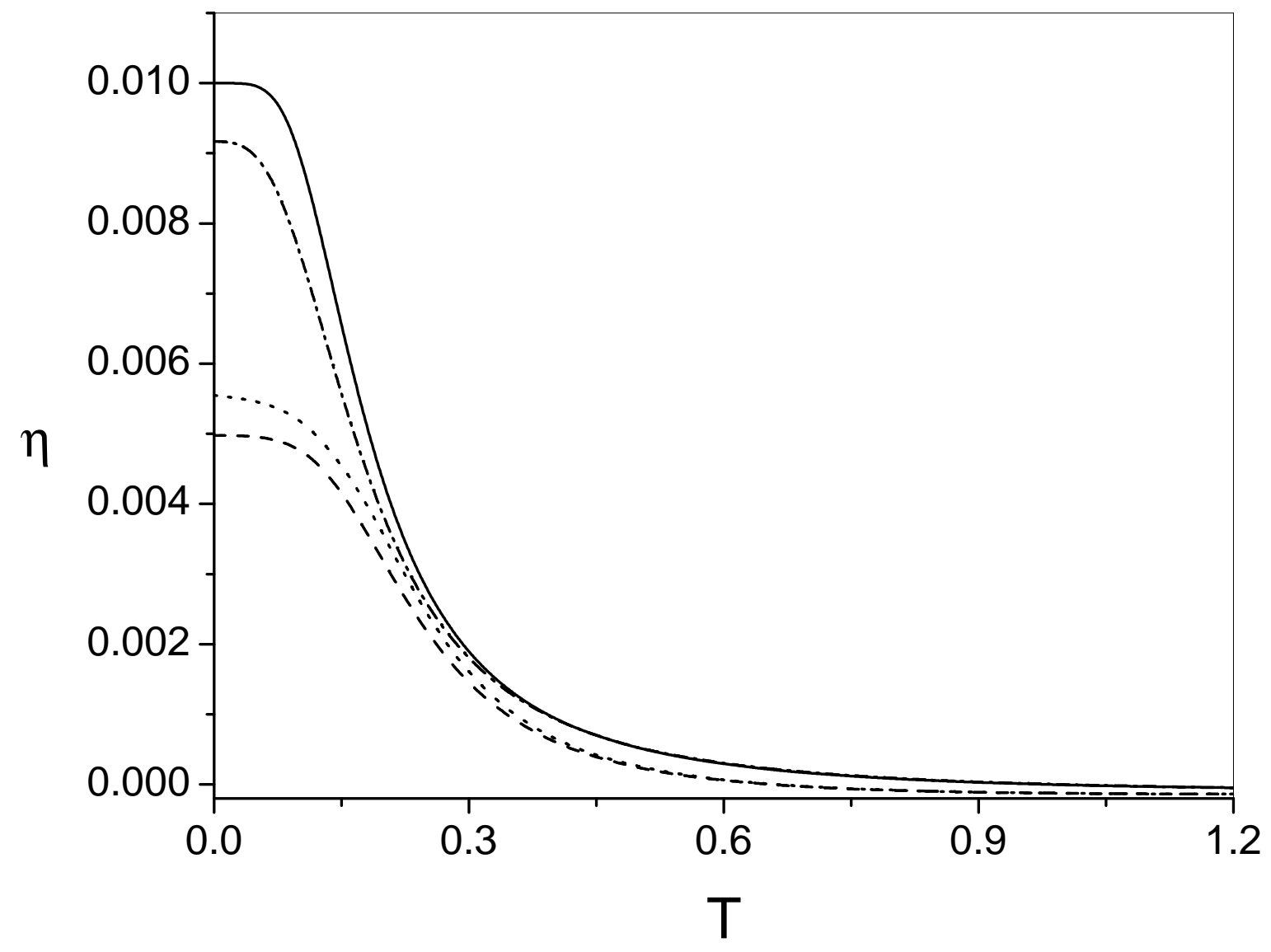

Fig.5 


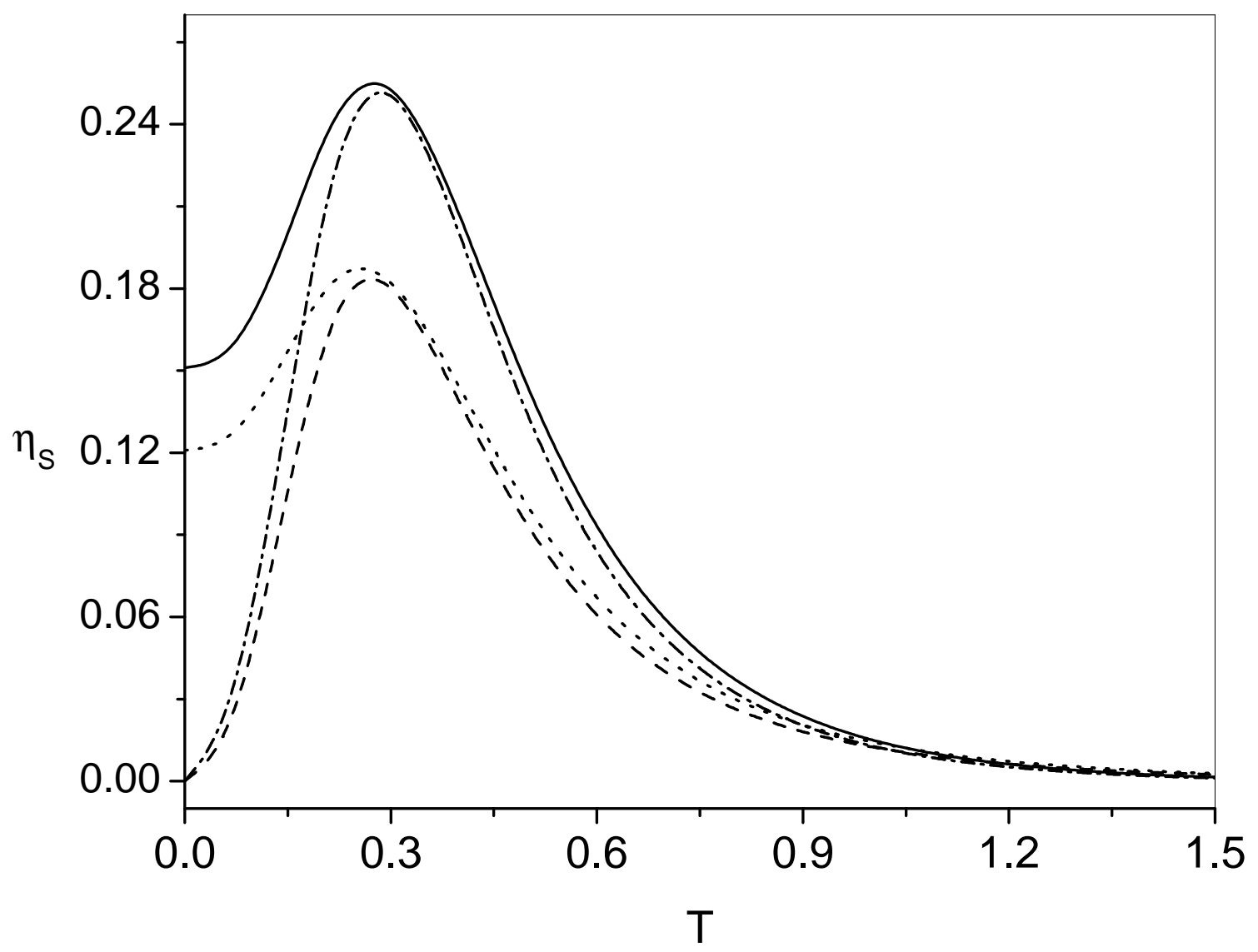

Fig.6 